\title{
Clinical outcome of radioiodine treatment for Graves' disease at a tertiary care centre
}

\author{
Rathore A, Mathew R, Garg A, Vanderpump M \\ Department of Endocrinology, Royal Free Hospital, London
}

\section{INTRODUCTION}

Radioiodine has been an effective modality of treatment for hyperthyroidism for over 70 years. There have been concerns over potential weight gain and exacerbation of thyroid orbitopathy although good quality evidence is lacking. We conducted a retrospective audit to look into the success rate and potential complications of radioiodine treatment in Graves' disease at our centre.

\section{Aims of the Audit:}

- To evaluate success rate of radioiodine treatment for Graves' disease at Royal Free Hospital, London in a retrospective analysis.

- To establish frequency of complications

\section{Methodology}

- Patients were identified following a search of the nuclear medicine departmental database.

- Consecutive patients who had radioiodine in three year period from January 2009 to December 2011 were included in the audit.

- Information was obtained from medical notes, pathology and nuclear medicine database. Data was analysed using Microsoft Excel.

\section{Demographics}

- 50 patients (34\% men; 66\% women) had radioiodine for Graves' disease over 3 year period. The mean age was 47 years (27-90).

- Four patients (8\%) had previously received a dose of radioiodine.

- $91 \%$ had received anti-thyroid drugs using a titration regime. None had a previous thyroidectomy.

- After radioiodine, $30 \%$ required further treatment with anti-thyroid drugs

\section{Success rate}

- At two-year follow-up, $86 \%$ had developed hypothyroidism, one patient remained euthyroid and $12 \%$ remained hyperthyroid.

- Among the 4 patients who were receiving a second dose of radioiodine, three became hypothyroid and one remained hyperthyroid.

- The average time of diagnosis of hypothyroidism was 5 months post radioiodine (2 -11.5 months)

\section{Complications}

- At 12 month follow-up, $69 \%$ of patients had gained weight and $31 \%$ had lost weight. Overall there was an average weight gain of $2.65 \mathrm{~kg}$. Men had slightly more average weight gain than women $(3.72 \mathrm{~kg}$ vs $2.17 \mathrm{~kg})$. Patients over 50 years of age had a greater average weight gain compared to those aged 50 or less $(4.01 \mathrm{~kg}$ vs $1.06 \mathrm{~kg})$. Fig 1 and 2 illustrate individual body weight changes at 6 and 12 months' follow up respectively.

- Eleven patients had pre-existing orbitopathy of which seven patients received steroid prophylaxis. No exacerbation of orbitopathy was reported. One case of new orbitopathy was diagnosed on follow-up where only lubricating eye drops were required.

- Thyroiditis was diagnosed in three patients. No other complications were noted.
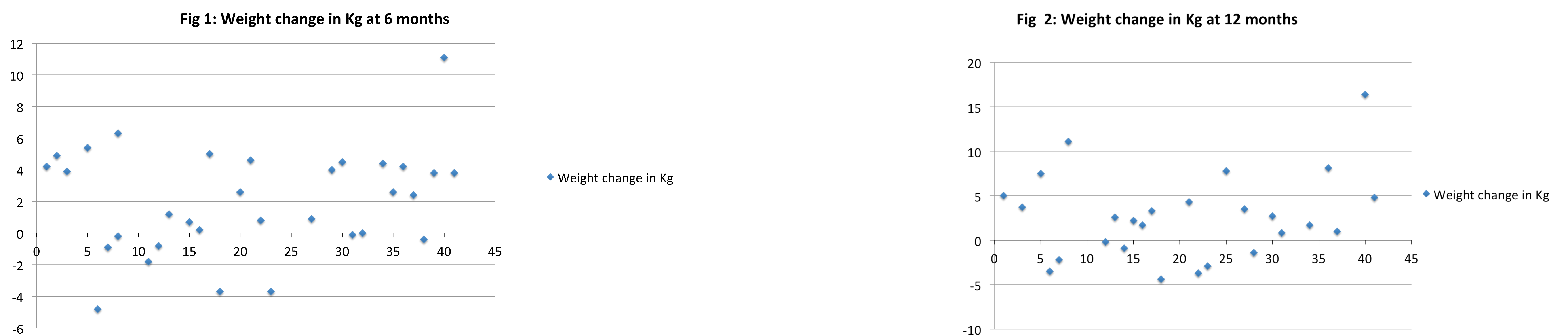

\section{Conclusions}

- Radioiodine treatment for Graves' disease has been proven to be an effective and safe treatment modality.

- The overall success rate in achieving hypothyroidism or euthyroidism was $88 \%$.

- Weight gain was variable. In general, male gender and older age were associated with greater weight gain.

- There was no exacerbation of pre-existing orbitopathy despite variations in practice regarding steroid prophylaxis. 